\title{
Aerobic Responses to FES-Assisted and Volitional Cycling in Children with Cerebral Palsy
}

\author{
Ashwini Sansare ${ }^{1,2}$, Ann Tokay Harrington ${ }^{3}$, Henry Wright ${ }^{2}$, James Alesi ${ }^{2}$, Ahad Behboodi ${ }^{4}$, \\ Khushboo Verma ${ }^{1,2}$ and Samuel C. K. Lee ${ }^{1,2, *(\mathbb{D})}$ \\ 1 Biomechanics and Movement Science Program, University of Delaware, Newark, DE 19713, USA; \\ ashwini@udel.edu (A.S.); vkhush@udel.edu (K.V.) \\ 2 Department of Physical Therapy, University of Delaware, Newark, DE 19713, USA; henryw@udel.edu (H.W.); \\ jfalesi@udel.edu (J.A.) \\ 3 Department of Physical Therapy, Arcadia University, Glenside, PA 19038, USA; harringtona@arcadia.edu \\ 4 Functional and Applied Biomechanics Section, Rehabilitation Medicine Department, \\ National Institutes of Health, Bethesda, MD 20892, USA; ahad.behboodi@nih.gov \\ * Correspondence: slee@udel.edu; Tel.: +1-302-831-2450
}

Citation: Sansare, A.; Harrington, A.T.; Wright, H.; Alesi, J.; Behboodi, A.; Verma, K.; Lee, S.C.K. Aerobic Responses to FES-Assisted and Volitional Cycling in Children with Cerebral Palsy. Sensors 2021, 21, 7590 https://doi.org/10.3390/s21227590

Academic Editor:

Benedicte Vanwanseele

Received: 29 September 2021

Accepted: 11 November 2021

Published: 15 November 2021

Publisher's Note: MDPI stays neutral with regard to jurisdictional claims in published maps and institutional affiliations.

Copyright: (c) 2021 by the authors. Licensee MDPI, Basel, Switzerland. This article is an open access article distributed under the terms and conditions of the Creative Commons Attribution (CC BY) license (https:/ / creativecommons.org/licenses/by/ $4.0 /)$.

\begin{abstract}
Recumbent stationary cycling is a potential exercise modality for individuals with cerebral palsy $(\mathrm{CP})$ that lack the postural control needed for upright exercises. Functional electrical stimulation (FES) of lower extremity muscles can help such individuals reach the cycling intensities that are required for aerobic benefits. The aim of this study was to examine the effect of cycling with and without FES assistance to that of a no-intervention control group on the cardiorespiratory fitness of children with CP. Thirty-nine participants were randomized to a FES group that underwent an 8-week FES-assisted cycling program, the volitional group (VOL), who cycled without FES, or a no-intervention control group (CON) (15 FES, $11 \mathrm{VOL}, 13 \mathrm{CON})$. Cadence, peak $\mathrm{VO}_{2}$, and net rise in heart rate were assessed at baseline, end of training, and washout (8-weeks after cessation of training). Latent growth curve modeling was used for analysis. The FES group showed significantly higher cycling cadences than the VOL and CON groups at POST and WO. There were no differences in improvements in the peak $\mathrm{VO}_{2}$ and peak net HR between groups. FES-assisted cycling may help children with $\mathrm{CP}$ attain higher cycling cadences and to retain these gains after training cessation. Higher training intensities may be necessary to obtain improvements in peak $\mathrm{VO}_{2}$ and heart rate.
\end{abstract}

Keywords: cardiorespiratory fitness; recumbent cycling; tricycle; biking

\section{Introduction}

Individuals with cerebral palsy $(\mathrm{CP})$ present with reduced muscle strength, muscle tone abnormalities, co-contraction of agonist and antagonist muscles, and poor selective voluntary motor control [1,2]. These aforementioned impairments result in poor motor performance and reduced mobility in children with CP. As children with CP enter adolescence and adulthood, there is a decline in independence, limited participation in physical activities and sports, and poor cardiorespiratory fitness compared to their peers [3-5]. Furthermore, limited accessibility to community fitness resources, recreational activities, exercise equipment, and lack of appropriate physical education programs in schools form additional barriers to engaging in a physically active lifestyle [3,5-7]. Thus, there is a critical need to identify and develop safe and effective means of improving and maintaining physical fitness levels in children with CP.

Recumbent cycling is a potential exercise modality for individuals lacking the strength or postural control necessary for exercises in standing or walking positions [8,9]. Abnormal muscle tone and motor activation, however, may prevent individuals with CP from attaining cycling cadences and heart rates required to produce aerobic benefits. Functional electrical stimulation (FES), which involves appropriately timed electrical muscle 
contractions for activity, may help such individuals achieve that cycling intensities that are necessary for aerobic benefits that are otherwise unachievable on their own. FES-assisted cycling improved fitness, bone mineral density, and muscle mass in individuals with spinal cord injuries [10-14]. In children with $\mathrm{CP}$, several case studies have shown improved cadence, power output, heart rate, and muscle strength and reduced co-contraction and oxygen expenditure following FES training [15-17]. In a recent randomized control trial (RCT), Armstrong et al. showed that an FES-based cycling combined with goal-directed training improved gross motor function and self-reported goal performance and satisfaction [18]. However, there are no larger RCTs that have assessed the effect of FES cycling on cardiorespiratory measures. Additionally, it is not known if FES assistance may provide cardiorespiratory benefits over and above a cycling-only protocols since these benefits could be attributed to improved fitness due to cycling and not FES-assistance.

The aim of this study was to examine the effect of two training approaches, cycling with and without FES assistance, to that of a no-intervention control group on the cardiorespiratory fitness of children with CP. Specifically, this study investigated group differences between participants undergoing an FES-assisted cycling training protocol (FES group), participants undergoing volitional cycling only (VOL group), and participants receiving no intervention (CON group) on cadence, peak oxygen uptake (peak $\mathrm{VO}_{2}$ ), and heart rate (HR) measures (1) following 8-weeks of cycling training and (2) after an 8-week washout period to assess the retention of the training effects. The hypothesis was that the FES group would show the greatest increase in cadence, peak $\mathrm{VO}_{2}$, and heart rate following the intervention period followed by VOL and lastly by CON. Additionally, it was hypothesized that the FES group would show the greatest retention in their peak $\mathrm{VO}_{2}$, heart rate, and cadence gains after a washout period followed by VOL and lastly by CON.

\section{Materials and Methods}

\subsection{Study Design}

A parallel three-group, randomized, cross-sectional experimental design was used. Based on previously collected pilot data, an a priori power analysis determined a sample size of 60 subjects, with approximately 20 subjects per group needed to produce significant results. Appropriate institutional review board permissions were obtained. All study procedures were explained, and all participants signed informed assent or consent documents (if 18 years old), and a parent or legal guardian signed consent documents for minors.

\subsection{Participants}

Children and young people with spastic diplegic $\mathrm{CP}$ between the ages of 10-18 years were recruited from the outpatient CP clinic at Shriners Hospitals for Children, Philadelphia, and local referral sources. All individuals were screened by a physical therapist and an orthopedic surgeon for the inclusion and exclusion criteria (Table 1) and were randomized to one of three groups: FES, VOL, or CON. Block randomization was used to ensure equal allocation across the three groups. Sequentially numbered, opaque, sealed envelopes in block sizes of three, with one envelope each for FES, VOL, and CON in random order within each block, were opened after participant consent was obtained. The participants and the research team were not blinded. 
Table 1. Inclusion and exclusion criteria.

\begin{tabular}{|c|c|}
\hline Inclusive Criteria & Exclusive Criteria \\
\hline $\begin{array}{l}\text { Age } 10-18 \text { years. } \\
\text { Gross Motor Function Classification Scale II, III, } \\
\text { or IV. } \\
\text { Adequate range of motion of the hips, knees, } \\
\text { and ankles to allow pedaling. } \\
\text { Self-reported visuo-perceptual skills and } \\
\text { cognitive/communication skills to follow } \\
\text { multiple step commands for attending to } \\
\text { exercise and data collection. } \\
\text { Ability to communicate pain or discomfort } \\
\text { with testing and training procedures. }\end{array}$ & $\begin{array}{l}\text { Lower-extremity orthopedic surgery or } \\
\text { traumatic fracture within the past } 6 \text { months. } \\
\text { Lower-extremity joint pain during cycling. } \\
\text { Hip, knee, or ankle joint instability or } \\
\text { dislocation. } \\
\text { Lower-limb stress fractures in the past year. } \\
\text { Symptomatic or current diagnosis of cardiac } \\
\text { disease as assessed by the American Heart } \\
\text { Association guidelines for cardiac history. } \\
\text { Current pulmonary disease or asthma and } \\
\text { taking oral steroids or hospitalized for an acute } \\
\text { episode in the past } 6 \text { months. } \\
\text { Severe spasticity in legs (score of } 4 \text { on the } \\
\text { Modified Ashworth Scale). } \\
\text { Severely limited joint range of motion or } \\
\text { irreversible muscle contractures that prevented } \\
\text { safe positioning on the cycle. }\end{array}$ \\
\hline
\end{tabular}

\subsection{Instrumentation}

Participants in the FES and VOL groups were trained on commercial recumbent sport tricycles (KMXKart, Birmingham, UK) instrumented with sensors to enable the calculation of the cycling power output [15]. The tricycles were mounted on a stationary trainer, and those receiving FES assistance included a tricycle mounted FES stimulator. The tricycle crank and spindle assembly were instrumented with a torque sensor and encoder to measure the torque applied during each pedal stroke, calculate power output, and to indicate crank position and cadence. During testing assessments, a similarly instrumented tricycle was used. For the FES group, a RehaStim stimulator (Hasomed GmbH, Magdeburg, Germany) was controlled by custom software (The Math Works Inc., MATLAB) to apply stimulation to the bilateral quadriceps femoris muscles. FES was applied at a current of $40 \mathrm{~mA}$ and a frequency of $50 \mathrm{~Hz}$ via transcutaneous electrodes while the FES software modulated the stimulus pulse duration. The quadriceps muscle was stimulated (via MATLAB Simulink control) in coordination with the crank angle of the tricycle during the "pushing phase", i.e., the phase during which the hip and knee went from maximum flexion to moderate hip-knee extension (the arc of cycling motion when the pedal was located between $\sim 40$ degrees before and $\sim 70$ degrees past top dead center, respectively).

\subsection{Procedure}

\subsubsection{Set-Up Phase}

Because recumbent cycling was a novel activity for the children with $\mathrm{CP}$, all of the participants underwent a set-up/practice phase prior to baseline testing (three $20 \mathrm{~min}$ cycling sessions in a laboratory setting). For both of the treatment groups, the participants and their caregivers were trained on system setup and how to implement the cycling training protocol at home. For the FES group, the participants practiced cycling with a gradual increase in the intensity of the stimulation until the maximum tolerable pulse duration was achieved. The pulse durations corresponding to the sensory perception of the stimulation and the maximum tolerable intensity were recorded and entered into the individual's custom FES cycling program.

An incremental cycling exercise test was performed during the initial visit to determine the power output range that corresponded with the $50-80 \%$ Karvonen target heart rate (details of which are under the section on Outcome Measures). This range of power output was later used as a target to guide the cycling intensity during the intervention phase for FES and VOL groups via visual feedback displayed on a laptop. Cadence, peak $\mathrm{VO}_{2}$, and peak net rise in heart rate were all obtained during the incremental cycle test. 
The participants from the FES group were sent home with an FES tricycle system and a Polar heart rate monitor (E600, Polar Electro, Kempele, Finland), while those in the VOL group were sent home with the same systems excluding the stimulator. A home-training $\log$ was maintained by each family. For both cycling groups, percentage adherence was calculated by dividing the number of sessions logged during the training period by the total expected number of sessions (24 sessions).

\subsubsection{Training Protocol FES Group}

The participants were asked to cycle continuously for $30 \mathrm{~min}$ three times a week for 8 weeks at the target cycling power corresponding to $50-80 \%$ of their Karvonenpredicted target heart rate during the baseline incremental test. Electrodes were placed over the quadriceps muscles of both legs and the stimulation settings for FES determined during the set-up phase were used. Exercise was performed while using a video game graphic on a laptop to help encourage the maintenance of the prescribed power output. The video game consisted of an airplane whose vertical height was controlled by the cycling power generated by the participant; the center of the screen corresponded to the participant's specific target cycling power. The participant was prompted to cycle and to maintain the airplane in the center of the screen. If the participant was unable to attain the target power, the FES-stimulation ramped from the sensory level (the pulse duration that produced cutaneous sensation) to a motor level that assisted the individual in maintaining the targeted power output. Maximum stimulation output was limited to the pulse duration corresponding to the participant's maximum tolerable level. If the participant cycled more than prescribed, then the stimulation ramped down until the prescribed power level was reached. If the participant was able to maintain the prescribed output, then the stimulation ramped down to sensory levels. If the participants could not initially cycle continuously for $30 \mathrm{~min}$ when they started the cycling protocol, they were asked to cycle for as long as possible, with brief rest breaks until a total exercise time of $30 \mathrm{~min}$ was attained. If a participant missed a training session during the week, make up sessions were allowed as long as the sessions did not exceed 4 times per week.

\section{VOL Group}

This group used the same cycling setup as the FES group but did not receive any stimulation during training. They also followed the same cycling protocol of $30 \mathrm{~min}$ three times a week for 8 weeks while maintaining a 50-80\% Karvonen-predicted target heart rate zone.

\section{CON Group}

This group did not participate in any cycling intervention during the 8-week period.

\subsection{Outcome Measures}

Assessments of cardiorespiratory fitness were conducted for all three groups at three time points: prior to training (PRE), at the end of 8 weeks of training (POST), and during a washout period of 8 weeks after the cessation of the training protocol (WO). An additional assessment was performed midway through training to account for increased cardiorespiratory capacity and motor learning effects, and new HR and power targets were set.

The outcome measures included: (a) cadence (rpm, number of cycling revolutions completed in one minute), (b) peak $\mathrm{VO}_{2}$ (liters of oxygen per minute per $\mathrm{kg}$ body weight), and (c) peak net HR (peak HR in beats per minute (bpm) during exercise-resting HR). Resting baseline measurements of peak $\mathrm{VO}_{2}$ and heart rate were taken for five minutes. Each participant's maximum power output and mean cadence was determined from an all-out pedaling trial, where the participants pedaled as hard and as fast as they could for one minute, typically performed the day before to avoid fatigue prior to the subsequent 
incremental load test. For the incremental load test, participants first performed a "warmup" phase. They sat relaxed in the seat and did not pedal actively while the auxiliary motor turned the crank passively at the mean cadence of the all-out pedaling trial. At the end of the first minute the motor was turned off and the participants began the "exercise phase" of pedaling actively while watching the visual feedback animation on the laptop to maintain the target power output. The power targets started at $10 \%$ of their maximum power from the all-out trial and increased by $10 \%$ every minute, until the subject was no longer able to maintain the power output target, became too tired to continue, or exceeded a respiratory equivalent ratio greater than 1.0. Following the exercise phase, participants completed a $1 \mathrm{~min}$ cool down period of passive cycling with the motor on followed by a $5 \mathrm{~min}$ recovery period. During the test, cadence, $\mathrm{HR}$, and breath by breath $\mathrm{VO}_{2}$ measurements were recorded and subsequently analyzed. An increase in these measures indicated an improvement in cardiorespiratory fitness.

\subsection{Statistical Analysis}

Two individuals in the FES group were lost at follow-up due to sickness or inability to come for assessment. Both $\mathrm{VO}_{2}$ and $\mathrm{HR}$ data were smoothed using a moving average window of $15 \mathrm{~s}$, and peak values were then selected. For computing the peak net rise in HR, resting HR on the day of testing was subtracted from the peak HR. Cadence was computed as the number of complete revolutions during the incremental test divided by time after excluding the warm-up and cool-down revolutions.

Analyses were conducted using a latent growth curve model (LGCM) in Mplus, (version 8.5, Muthe'n and Muthe'n, Los Angeles, CA, USA) [19]. LGCM is a growth curve analysis that models how individual trajectories of change over time (slope) differ for different groups of individuals to examine changes in an outcome measure over time. Traditional analyses of mean change (e.g., repeated measures ANOVA) examine the difference in the group means (and not the slope or the growth trajectory) at different time points; more importantly, they only use those participants in the analysis who have data across all the time points. This results in loss of information from participants with missing data at one or more time points, a highly likely scenario during a 16-week long study. LGCM, by using a maximum likelihood estimation for handling missing data, uses all of the participants in the analysis. It is thus more robust to partially missing data and unequally spaced time points; hence, it was the method of choice for data analysis.

Between group differences for FES, VOL, and CON were assessed on the withinperson change, i.e., the slope or the growth curves between PRE to POST and POST to WO for cadence, peak $\mathrm{VO}_{2}$, and peak net HR. The loadings of the intercept (starting point) factors in the model were not fixed, allowing each participant to have their own initial level of cadence, peak $\mathrm{VO}_{2}$, and peak net $\mathrm{HR}$. The residual variances were freely estimated for all three outcome measures. Based on the recommendations by Feingold [20], effect sizes were estimated at the POST and WO by dividing the difference between the experimental and control group mean growth rates by the standard deviation at baseline. The resultant effect sizes that were produced for a growth curve model $\left(\mathrm{d}_{\mathrm{GMA}}\right)$ were analogous to those generated by the traditional methods using Cohen's $\mathrm{d}$ with the following interpretation criteria: small (0.20), moderate (0.50), and large (0.80).

\section{Results}

Figure 1 summarizes the trial flowchart for this study. Thirty-nine participants enrolled in the study (15 FES, 11 VOL, 13 CON). The patient characteristics at baseline (Table 2) showed no significant differences in age, height, weight, and BMI among the three groups on one-way ANOVA ( $p=0.300, p=0.389, p=0.081, p=0.133$, respectively). The average adherence to the training protocol in both the cycling groups was $91.9 \%$, with no significant difference between the FES and VOL groups $(p=0.118)$. 


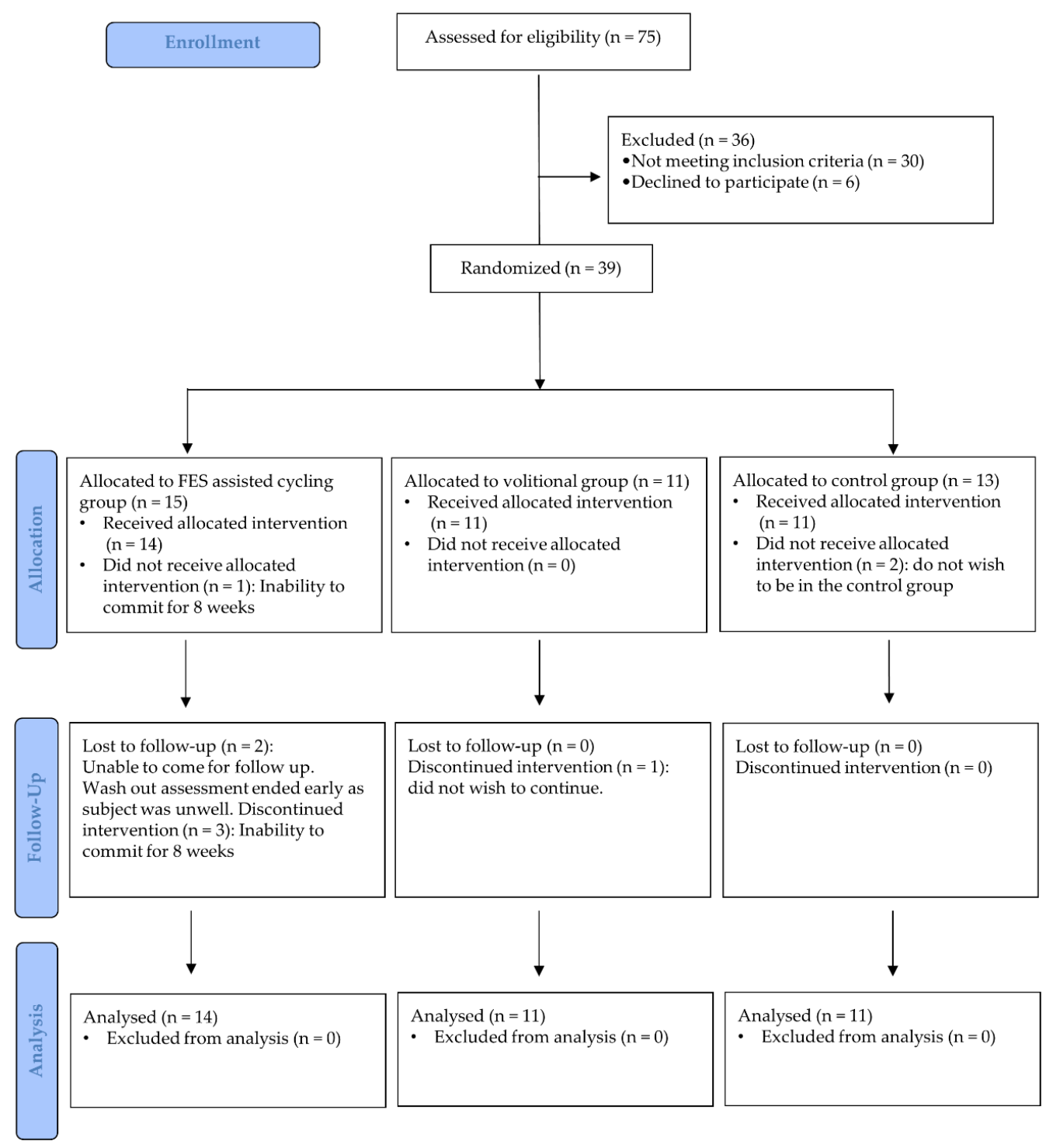

Figure 1. Participant flow diagram illustrating the number of participants who were randomly assigned, received the intended treatment, and analyzed for the cardiovascular outcomes for each group.

Table 2. Baseline characteristics of participants. Average age, height, mass, and BMI differences (mean \pm SD) between the three groups: FES cycling group, volitional group, and the control group.

\begin{tabular}{|c|c|c|c|}
\hline Participant Characteristics & FES Cycling $(n=14)$ & Volitional $(n=11)$ & Control $(n=11)$ \\
\hline \multicolumn{4}{|l|}{ Demographics } \\
\hline Age, $y$ & $14.5(2.4)$ & $12.7(2.1)$ & $13.7(2.9)$ \\
\hline Males & 13 & 8 & 9 \\
\hline \multicolumn{4}{|l|}{ Anthropometrics } \\
\hline Height, $\mathrm{m}$ & $1.53(0.14)$ & $1.43(0.14)$ & $1.51(0.19)$ \\
\hline Mass, kg & $56.49(22.6)$ & $38.08(12.8)$ & $47.05(17.4)$ \\
\hline BMI, $\mathrm{kg} / \mathrm{m}^{2}$ & $23.81(6.2)$ & $18.63(3.8)$ & $20.85(8.0)$ \\
\hline \multicolumn{4}{|l|}{ GMFCS level } \\
\hline II & 6 & 2 & 4 \\
\hline III & 3 & 4 & 4 \\
\hline IV & 5 & 5 & 3 \\
\hline
\end{tabular}

BMI, body mass index; GMFCS, gross motor function classification system; FES, functional electrical stimulation. 
Table 3 depicts the descriptive statistics, including the means and 95\% confidence intervals, for cadence, peak $\mathrm{VO}_{2}$, and peak net $\mathrm{HR}$ across PRE, POST, and WO. The results of the LGCM that estimated the slope of the outcome measures across PRE to POST and POST to WO time points (Figure 2) are explained for each outcome below:

Table 3. Means and 95\% confidence intervals at PRE, POST, and $\mathrm{WO}$ for cadence, peak $\mathrm{VO}_{2}$, and peak net $\mathrm{HR}$.

\begin{tabular}{|c|c|c|c|c|c|c|c|c|c|c|}
\hline & \multirow{3}{*}{$\begin{array}{c}\text { Group } \\
\text { FES }\end{array}$} & \multicolumn{3}{|c|}{ PRE } & \multicolumn{3}{|c|}{ POST } & \multicolumn{3}{|c|}{ WO } \\
\hline & & \multirow{2}{*}{$\begin{array}{c}\text { Mean } \\
44\end{array}$} & \multicolumn{2}{|c|}{$95 \% \mathrm{CI}$} & \multirow{2}{*}{$\begin{array}{c}\text { Mean } \\
46\end{array}$} & \multicolumn{2}{|c|}{$95 \%$ CI } & \multirow{2}{*}{$\begin{array}{c}\text { Mean } \\
48\end{array}$} & \multicolumn{2}{|c|}{$95 \% \mathrm{CI}$} \\
\hline \multirow{3}{*}{ Cadence (rev/min) } & & & 33 & 55 & & 44 & 49 & & 47 & 52 \\
\hline & VOL & 33 & 22 & 45 & 35 & 32 & 37 & 35 & 31 & 39 \\
\hline & $\mathrm{CON}$ & 42 & 34 & 50 & 42 & 40 & 43 & 42 & 39 & 45 \\
\hline \multirow{3}{*}{ Peak $\mathrm{VO}_{2}(\mathrm{~mL} / \mathrm{kg} / \mathrm{min})$} & FES & 24.9 & 18.4 & 31.4 & 26.2 & 23.4 & 28.9 & 24.8 & 20.6 & 29 \\
\hline & VOL & 24.5 & 17.7 & 31.4 & 25.3 & 22.5 & 28.1 & 26.9 & 22.9 & 31 \\
\hline & $\mathrm{CON}$ & 24.9 & 20 & 29.9 & 24.4 & 22.4 & 26.4 & 25 & 22.1 & 27.9 \\
\hline \multirow{3}{*}{ Peak Net HR (beats/min) } & FES & 61 & 45 & 77 & 63 & 56 & 69 & 62 & 49 & 74 \\
\hline & VOL & 47 & 30 & 64 & 52 & 45 & 59 & 56 & 44 & 68 \\
\hline & $\mathrm{CON}$ & 49 & 37 & 61 & 52 & 48 & 57 & 49 & 41 & 57 \\
\hline
\end{tabular}

FES, functional electrical stimulation; $\mathrm{VOL}$, volitional; $\mathrm{CON}$, control; $\mathrm{WO}$, washout; $\mathrm{VO}_{2}$, ventilated oxygen; $\mathrm{HR}$, heart rate.

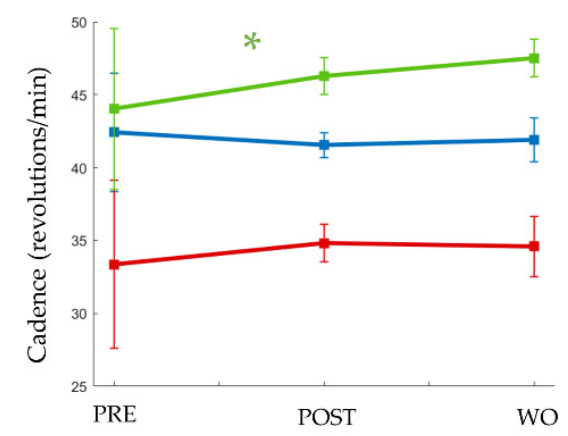

(a)

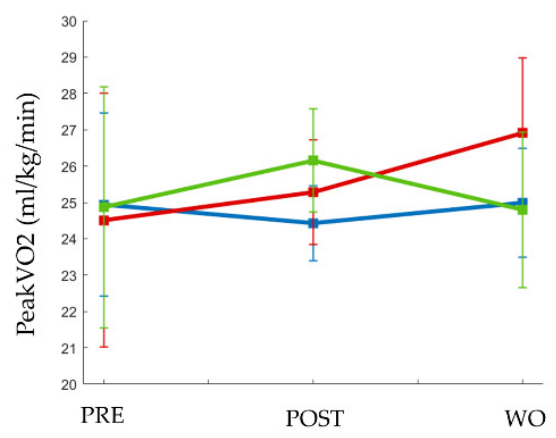

(b)

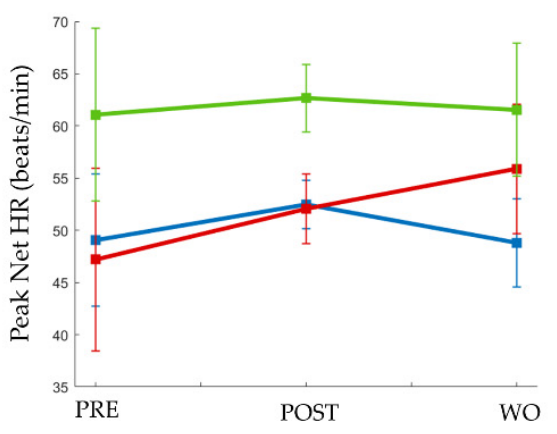

(c)

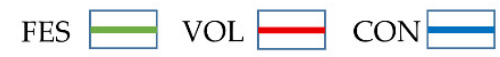

Figure 2. Cardiovascular performance during incremental test in the functional electrical stimulation-assisted cycling (FES), volitional cycling (VOL), and control (CON) groups. The graphs depict the slope of the (a) cadence, (b) peak $\mathrm{VO}_{2}$, and (c) peak net HR across PRE to POST and POST to washout (WO) time points. Error bars depict standard error of the mean at the three time points.

\subsection{Cadence}

The results of the model show that over 8 weeks of training from PRE to POST, the $\mathrm{CON}$ group experienced a decline in cadence $(\mathrm{B}=-0.865, p=0.402)$, while the VOL group experienced a small increase $(B=1.455, p=0.204)$, both of which were not significant. Conversely, the FES group experienced a statistically significant increase at POST (B $=2.238$, $p=0.041)$, which was also significantly higher compared to the CON group $(\mathrm{B}=3.104$, $p=0.039$ ). The effect size for the difference between FES and CON group was small $\left(\mathrm{d}_{\mathrm{GMA}}=0.4\right)$. The increase in the VOL group was not statistically significantly different from the increase observed in the FES group $(p=0.622)$ and the slight decrease observed in the CON group $(p=0.132)$. The effect size for the difference between VOL and CON was small $\left(\mathrm{d}_{\mathrm{GMA}}=0.3\right)$.

At the end of the washout period, all three groups (CON: $\mathrm{B}=0.343, p=0.840$; VOL: $\mathrm{B}=-0.210, p=0.903$; FES: $\mathrm{B}=1.244, p=0.487$ ) showed minimal changes in the slope of the mean cadence between POST to WO. Therefore, the slope of the cadence line between POST to WO changed very little, i.e., it stayed relatively horizontal. Since VOL and CON did not make significant gains between PRE to POST, a minimal change in 
the slope between POST to WO indicated that they maintained the same status quo over WO as well. However, a "flat" line between POST to WO for the FES group implied that the gains between PRE to POST were retained in the POST to WO period. There were no statistically significant differences between the change in the VOL and FES group $(p=0.559)$ and in the VOL and CON group $(p=0.819)$. The effect sizes for the difference between FES and CON $\left(\mathrm{d}_{\mathrm{GMA}}=0.1\right)$ and for the difference between VOL and CON $\left(\mathrm{d}_{\mathrm{GMA}}<0.1\right)$ were negligible.

\subsection{Peak $\mathrm{VO}_{2}$}

Over the 8 weeks of training, the CON group experienced a decline $(B=-0.502$, $p=0.681)$, while both the VOL $(\mathrm{B}=0.764, p=0.528)$ and FES $(\mathrm{B}=1.279, p=0.276)$ groups showed an increase from PRE to POST, with slope differences that were not statistically significant. Small effect sizes were found for difference between FES and CON $\left(\mathrm{d}_{\mathrm{GMA}}=0.4\right)$ and between VOL and CON $\left(\mathrm{d}_{\mathrm{GMA}}=0.3\right)$. Over the 8 weeks of the washout period, the CON $(B=0.558, p=0.753)$ and VOL $(B=1.630, p=0.339)$ groups experienced a minimal increase, while the FES group experienced a minimal decrease $(B=-1.349, p=0.455)$, neither of which were statistically significant. The effect size for the difference between FES and CON was small $\left(\mathrm{d}_{\mathrm{GMA}}=0.2\right)$, and for difference between VOL and CON $\left(\mathrm{d}_{\mathrm{GMA}}=0.1\right)$, it was negligible.

\subsection{Peak Net HR}

Over the 8 weeks of training from PRE to POST, all three groups (CON: B $=3.415$, $p=0.217$; VOL: $\mathrm{B}=4.821, p=0.095$, FES: $\mathrm{B}=1.662, p=0.535$ ) showed a minimal increase that was not statistically significant. The effect sizes for the difference between FES and CON $\left(\mathrm{d}_{\mathrm{GMA}}=0.1\right)$ and for the difference between VOL and CON $\left(\mathrm{d}_{\mathrm{GMA}}=0.1\right)$ were negligible.

Over the 8 weeks of the washout period, the CON group $(B=-3.669, p=0.467)$ and FES groups $(B=-1.242, p=0.826)$ demonstrated a decline, while the VOL group experienced an increase $(B=3.842, p=0.475)$ from POST to WO that was not significant. The effect size for the difference between FES and CON was negligible $\left(\mathrm{d}_{\mathrm{GMA}}=0.1\right)$, and that for the difference between VOL and CON was small $\left(\mathrm{d}_{\mathrm{GMA}}=0.3\right)$.

For all three outcome measures across all time points, covariance was not statistically significant, indicating that the initial level of the cadence, peak $\mathrm{VO}_{2}$, and $\mathrm{HR}$ was not related to the rate of change (slope) in these measures.

\section{Discussion}

This RCT investigated the benefits of FES-assisted cycling and volitional cycling over a no-intervention control group on cardiorespiratory fitness in children with CP over an 8-week cycling training protocol. Additionally, this study also investigated the ability to retain the training effects on cardiorespiratory parameters after an 8-week washout period.

The findings from this study partially confirm the hypothesis and indicate that while FES-assisted cycling can enable children with CP to attain higher cycling cadences than a cycling alone protocol or without any intervention, it did not show any significant improvements in peak $\mathrm{VO}_{2}$ and peak net HR. Furthermore, all three groups showed minimal changes between POST to WO. It is important to note that the PRE-to-POST changes need to be taken into account while interpreting the results. Because the CON and VOL groups did not show significant changes between PRE to POST, a minimal change between POST to WO indicates that overall, across 8 weeks of training and 8 weeks after the cessation of training, the CON and VOL groups did not change much. However, because the FES group made significant gains between PRE to POST, a minimal change between POST to WO is desirable and is indicative of the ability to maintain the gains made during training. Hence, the results show that FES assistance helped retain the higher gains in cadence, even after the cessation of the training. Higher cadences are a result of improved muscle coordination and timing. FES training may have facilitated motor activation via the improved timing and intensity of muscle contractions. Thus, FES assistance may lead to 
improved functional movement patterns and pedaling efficiency. Additionally, for changes in the cadence from PRE to POST, the slope of VOL (i.e., the increase from PRE to POST) was not statistically significant from either the slope of the FES group or the CON group. This is because the increase in the VOL was small, and it fell between the higher increase in the FES group and the no increase in the CON group, i.e., the slope of the VOL lies between the more positive slope of the FES group and the slight negative slope of the $\mathrm{CON}$ group. Hence, it was not statistically significant from either group. This implies that cycling training without FES may help with attaining a higher cycling cadence compared to no training at all but is not as effective as adding FES assistance to cycling.

Our effect sizes also support that both FES and VOL showed a small effect for the cadence and peak $\mathrm{VO}_{2}$, with that of FES group being slightly higher than the VOL group. Increased cycling cadence should ideally lead to corresponding improvements in peak $\mathrm{VO}_{2}$ and peak net HR, which was not true for the cohort. Both the FES and VOL group showed slight improvements in peak $\mathrm{VO}_{2}$ that were not significant compared to $\mathrm{CON}$. For peak net HR, both the VOL and CON groups showed a slight increase, but FES remained relatively stable. This trend is reflected in the effect sizes for the VOL and FES groups at POST, which were small and negligible, respectively. A possible explanation might be that the participants in the VOL and FES groups were cycling at the $50-80 \%$ Karvonen maximum-predicted target HR during the at-home training phase. While it is standard practice to determine training intensities using a percentage of the Karvonen-predicted HRmax, a broad range such as $50-80 \%$ may have led to the participants training only at the lower end of this range, which may not have been enough to attain therapeutic benefits. Hence, using a narrower range such as the $70-80 \%$ Karvonen maximum predicted HR, which has been recommended as the threshold for cardiorespiratory training in young adults, may be more effective [21].

It is important to note that the current study was underpowered to find a statistically significant difference. A priori power analysis revealed a desired sample size of 60 , with 20 participants in each group. However, 36 participants were recruited overall $(\mathrm{N}=11$ for $\mathrm{CON}, \mathrm{N}=11$ for VOL, $\mathrm{N}=14$ for FES). Additionally, the cohort comprised of participants with different functional abilities (GMFCS levels II-IV) and high variability at baseline, which was confirmed by the large confidence intervals at baseline. This heterogeneity combined with a relatively small sample size may contribute to a lack of between group differences. High intersubject variability has been a problem in several previous RCTs on children with CP, leading to insignificant between group difference results on some [22-24] or all [25] outcome measures.

This study only stimulated the quadriceps muscle during cycling because it is the main agonist that drives the typical recumbent cycling motion and provides a simple stimulation protocol that the families could execute at home. Johnston et al., however, demonstrated that not only do children with CP use the hamstrings in addition to the quadriceps while cycling, but they also have a higher degree of agonist-antagonist co-contraction at the hip, knee, and ankle muscles [26,27]. Thus, an approach where only the quadriceps muscle is stimulated may not have been enough to change the abnormal motor control strategies that prevent children with CP from cycling more efficiently. An RCT by Armstrong et al. showed improved gross motor strength and self-reported measures of goal performance and satisfaction after undergoing an 8-week FES cycling program that stimulated hamstrings, gluteal, gastrocnemius, and tibialis anterior muscles in addition to quadriceps. Adopting a more comprehensive stimulation strategy that stimulates the hip, knee, and ankle muscles might be more beneficial.

\section{Conclusions}

Numerous studies have examined the effect of FES in populations such as those of patients who have experienced stroke and spinal cord injury. This study is the first to investigate the aerobic responses to FES assistance during the cycling motion in children with $\mathrm{CP}$, which can serve as a safe exercise modality for patients with a wide spectrum of 
functional and ambulatory abilities as well as an enjoyable physical activity for children. The findings indicate that FES assistance can help children with $\mathrm{CP}$ attain higher cycling cadences and can help to retain these gains in cadence after the cessation of training. Similar increases were not obtained in the peak $\mathrm{VO}_{2}$ and peak net rise in the heart rates, indicating that a minimum heart rate target of $50 \%$ of the Karvonen maximum predicted heart rate may be insufficient to attain cardiorespiratory benefits. Thus, higher training intensities may be necessary to obtain improvements in peak $\mathrm{VO}_{2}$ and heart rate. Overall, this study provided some support that FES-assisted cycling may facilitate motor gains such as increased cycling cadence in children with $\mathrm{CP}$ and may potentially help them attain better fitness levels.

Author Contributions: Conceptualization, A.S. and S.C.K.L.; methodology, A.S., A.T.H., H.W., J.A. and A.B.; software, A.S., J.A. and H.W.; formal analysis, A.S. and A.B.; investigation, A.S., A.B., H.W. and A.T.H.; writing—original draft preparation, A.S.; writing—review and editing, A.S., K.V, J.A., A.T.H., S.C.K.L. and A.B.; visualization, A.S. and K.V.; supervision, S.C.K.L.; project administration, S.C.K.L.; funding acquisition, S.C.K.L. All authors have read and agreed to the published version of the manuscript.

Funding: This research was supported by National Institute of Health, grant \#R01HD062588 and Shriners Hospitals for Children, Philadelphia, Grant \#8530. APC funding was waived by Sensors.

Institutional Review Board Statement: The study was conducted according to the guidelines of the Declaration of Helsinki and was approved by the Institutional Review Board of Temple University (protocol code 11659).

Informed Consent Statement: Informed assent or consent (if 18 years old) was obtained from all subjects involved in the study, and a parent or legal guardian signed consent documents for minors. Written informed consent has been obtained from the patient(s) to publish this paper.

Data Availability Statement: The data presented in this study are available upon request from the corresponding author.

Conflicts of Interest: The authors declare no conflict of interest. The funders had no role in the design of the study; in the collection, analyses, or interpretation of data; in the writing of the manuscript; or in the decision to publish the results.

\section{References}

1. Damiano, D.L.; Martellotta, T.L.; Sullivan, D.J.; Granata, K.P.; Abel, M.F. Muscle Force Production and Functional Performance in Spastic Cerebral Palsy: Relationship of Cocontraction. Arch. Phys. Med. Rehabil. 2000, 81, 895-900. [CrossRef] [PubMed]

2. Thelen, D.D.; Riewald, S.A.; Asakawa, D.S.; Sanger, T.D.; Delp, S.L. Abnormal Coupling of Knee and Hip Moments during Maximal Exertions in Persons with Cerebral Palsy. Muscle Nerve 2003, 27, 486-493. [CrossRef] [PubMed]

3. Rimmer, J.H. Exercise and Physical Activity in Persons Aging with a Physical Disability. Phys. Med. Rehabil. Clin. N. Am. 2005, 16, 41-56. [CrossRef] [PubMed]

4. Orlin, M.N.; Palisano, R.J.; Chiarello, L.A.; Kang, L.; Polansky, M.; Almasri, N.; Maggs, J. Participation in Home, Extracurricular, and Community Activities among Children and Young People with Cerebral Palsy. Dev. Med. Child. Neurol. 2010, $52,160-166$. [CrossRef]

5. Fowler, E.G.; Kolobe, T.H.; Damiano, D.L.; Thorpe, D.E.; Morgan, D.W.; Brunstrom, J.E.; Coster, W.J.; Henderson, R.C.; Pitetti, K.H.; Rimmer, J.H.; et al. Promotion of Physical Fitness and Prevention of Secondary Conditions for Children with Cerebral Palsy: Section on Pediatrics Research Summit Proceedings. Phys. Ther. 2007, 87, 1495-1510. [CrossRef]

6. Rimmer, J.H.; Riley, B.; Wang, E.; Rauworth, A. Accessibility of Health Clubs for People with Mobility Disabilities and Visual Impairments. Am. J. Public Health 2005, 95, 2022-2028. [CrossRef]

7. Rimmer, J.H. Physical Fitness Levels of Persons with Cerebral Palsy. Dev. Med. Child. Neurol. 2001, 43, 208-212. [CrossRef]

8. Hicks, A.L.; Martin Ginis, K.A.; Pelletier, C.A.; Ditor, D.S.; Foulon, B.; Wolfe, D.L. The Effects of Exercise Training on Physical Capacity, Strength, Body Composition and Functional Performance among Adults with Spinal Cord Injury: A Systematic Review. Spinal Cord 2011, 49, 1103-1127. [CrossRef]

9. Siebert, K.L.; DeMuth, S.K.; Knutson, L.M.; Fowler, E.G. Stationary Cycling and Children with Cerebral Palsy: Case Reports for Two Participants. Phys. Occup. Ther. Pediatr. 2010, 30, 125-138. [CrossRef]

10. Johnston, T.E.; Smith, B.T.; Oladeji, O.; Betz, R.R.; Lauer, R.T. Outcomes of a Home Cycling Program using Functional Electrical Stimulation or Passive Motion for Children with Spinal Cord Injury: A Case Series. J. Spinal Cord Med. 2008, 31, $215-221$. [CrossRef] [PubMed] 
11. Duffell, L.D.; Donaldson, N.d.N.; Perkins, T.A.; Rushton, D.N.; Hunt, K.J.; Kakebeeke, T.H.; Newham, D.J. Long-Term Intensive Electrically Stimulated Cycling by Spinal Cord-Injured People: Effect on Muscle Properties and their Relation to Power Output. Muscle Nerve 2008, 38, 1304-1311. [CrossRef] [PubMed]

12. Chen, S.; Lai, C.; Chan, W.P.; Huang, M.; Tsai, H.; Chen, J.J. Increases in Bone Mineral Density After Functional Electrical Stimulation Cycling Exercises in Spinal Cord Injured Patients. Disabil. Rehabil. 2005, 27, 1337-1341. [CrossRef]

13. Griffin, L.; Decker, M.J.; Hwang, J.Y.; Wang, B.; Kitchen, K.; Ding, Z.; Ivy, J.L. Functional Electrical Stimulation Cycling Improves Body Composition, Metabolic and Neural Factors in Persons with Spinal Cord Injury. J. Electromyogr. Kinesiol. 2009, 19, 614-622. [CrossRef]

14. Frotzler, A.; Coupaud, S.; Perret, C.; Kakebeeke, T.H.; Hunt, K.J.; Donaldson, N.d.N.; Eser, P. High-Volume FES-Cycling Partially Reverses Bone Loss in People with Chronic Spinal Cord Injury. Bone 2008, 43, 169-176. [CrossRef]

15. Harrington, A.T.; McRae, C.G.A.; Lee, S.C.K. Evaluation of Functional Electrical Stimulation to Assist Cycling in Four Adolescents with Spastic Cerebral Palsy. Int. J. Pediatr. 2012, 2012, 504387. [CrossRef] [PubMed]

16. Peri, E.; Ambrosini, E.; Pedrocchi, A.; Pagliano, E.; Riva, D.; Baranello, G.; Arnoldi Maria, T.; Foscan, M.; Corlatti, A.; Marchi, A.; et al. Volitional Cycling Augmented by Functional Electrical Stimulation in Hemiparetic Adolescents: A Case Series Study. J. Autom. Control. 2013, 21,37-42. [CrossRef]

17. Trevisi, E.; Gualdi, S.; De Conti, C.; Salghetti, A.; Martinuzzi, A.; Pedrocchi, A.; Ferrante, S. Cycling Induced by Functional Electrical Stimulation in Children Affected by Cerebral Palsy: Case Report. Eur. J. Phys. Rehabil. Med. 2012, 48, 135-145. [PubMed]

18. Armstrong, E.L.; Boyd, R.N.; Horan, S.A.; Kentish, M.J.; Ware, R.S.; Carty, C.P. Functional Electrical Stimulation Cycling, GoalDirected Training, and Adapted Cycling for Children with Cerebral Palsy: A Randomized Controlled Trial. Dev. Med. Child. Neurol. 2020, 62, 1406-1413. [CrossRef] [PubMed]

19. Muthén, L.K.; Muthén, B.O. MPlus User's Guide, 8th ed.; Muthén \& Muthén: Los Angeles, CA, USA, 2017.

20. Feingold, A. Effect Sizes for Growth-Modeling Analysis for Controlled Clinical Trials in the Same Metric as for Classical Analysis. Psychol. Methods 2009, 14, 43-53. [CrossRef] [PubMed]

21. Bar-Or, O.; Rowland, T.W. Pediatric Exercise Medicine: From Physiologic Principles to Health Care Application; Human Kinetics: Champaign, IL, USA, 2004; 501p.

22. Fowler, E.G.; Knutson, L.M.; Demuth, S.K.; Siebert, K.L.; Simms, V.D.; Sugi, M.H.; Souza, R.B.; Karim, R.; Azen, S.P.; Physical Therapy Clinical Research Network (PTClinResNet). Pediatric Endurance and Limb Strengthening (PEDALS) for Children with Cerebral Palsy using Stationary Cycling: A Randomized Controlled Trial. Phys. Ther. 2010, 90, 367-381. [CrossRef]

23. Dodd, K.J.; Taylor, N.F.; Graham, H.K. A Randomized Clinical Trial of Strength Training in Young People with Cerebral Palsy. Dev. Med. Child. Neurol. 2003, 45, 652-657. [CrossRef]

24. Liao, H.F.; Liu, Y.C.; Liu, W.Y.; Lin, Y.T. Effectiveness of Loaded Sit-to-Stand Resistance Exercise for Children with Mild Spastic Diplegia: A Randomized Clinical Trial. Arch. Phys. Med. Rehabil. 2007, 88, 25-31. [CrossRef] [PubMed]

25. Patikas, D.; Wolf, S.I.; Armbrust, P.; Mund, K.; Schuster, W.; Dreher, T.; Döderlein, L. Effects of a Postoperative Resistive Exercise Program on the Knee Extension and Flexion Torque in Children with Cerebral Palsy: A Randomized Clinical Trial. Arch. Phys. Med. Rehabil. 2006, 87, 1161-1169. [CrossRef] [PubMed]

26. Johnston, T.E.; Barr, A.E.; Lee, S.C. Biomechanics of Submaximal Recumbent Cycling in Adolescents with and without Cerebral Palsy. Phys. Ther 2007, 87, 572-585. [CrossRef]

27. Roy, S.; Alves-Pinto, A.; Lampe, R. Characteristics of Lower Leg Muscle Activity in Patients with Cerebral Palsy during Cycling on an Ergometer. BioMed Res. Int. 2018, 2018, 6460981. [CrossRef] [PubMed] 